\title{
Article \\ Metformin Reverses tmexCD1-toprJ1- and tet(A)-Mediated High-Level Tigecycline Resistance in K. pneumoniae
}

\author{
Xia Xiao ${ }^{1,2, \dagger}$, Quanmin Huan ${ }^{1, \dagger}$, Yanhu Huang ${ }^{1}$, Yuan Liu 1,2,3 ${ }^{(D)}$, Ruichao Li 1,2,3 ${ }^{D}$, Xilan Xu 4 \\ and Zhiqiang Wang 1,2,3,5,*(D)
}

1 College of Veterinary Medicine, Yangzhou University, Yangzhou 225009, China; xiaoxia@yzu.edu.cn (X.X.); huanquanmin@126.com (Q.H.); yuqi29595585@163.com (Y.H.); liuyuan2018@yzu.edu.cn (Y.L.); rchl88@yeah.net (R.L.)

2 Jiangsu Co-Innovation Center for Prevention and Control of Important Animal Infectious Diseases and Zoonoses, Yangzhou 225009, China

3 Institute of Comparative Medicine, Yangzhou University, Yangzhou 225009, China

4 Pizhou Animal Health Supervision Institute, Xuzhou 320300, China; xiaxian6629285@163.com

5 Institutes of Agricultural Science and Technology Development, Yangzhou 225009, China

* Correspondence: zqwang@yzu.edu.cn; Tel.: +86-51487979224; Fax: +86-51487972218

+ These authors contributed equally to this study.

check for

updates

Citation: Xiao, X.; Huan, Q.; Huang,

Y.; Liu, Y.; Li, R.; Xu, X.; Wang, Z.

Metformin Reverses tmexCD1-toprJ1and tet(A)-Mediated High-Level

Tigecycline Resistance in $K$.

pneumoniae. Antibiotics 2022, 11, 162.

https://doi.org/10.3390/

antibiotics11020162

Academic Editor: Khondaker

Miraz Rahman

Received: 22 December 2021

Accepted: 25 January 2022

Published: 27 January 2022

Publisher's Note: MDPI stays neutral with regard to jurisdictional claims in published maps and institutional affiliations.

Copyright: (C) 2022 by the authors. Licensee MDPI, Basel, Switzerland. This article is an open access article distributed under the terms and conditions of the Creative Commons Attribution (CC BY) license (https:// creativecommons.org/licenses/by/ $4.0 /)$.
Abstract: Tigecycline (TIG) is one of the last effective options against multidrug resistance bacteria. Recently, the RND (resistance-nodulation-division) efflux pump gene cluster, tmexCD1-toprJ1, and the tetracycline-efflux pump tet(A) mutation were reported to mediate high level resistance to TIG in clinically important pathogens, weakening the efficacy of TIG. In this study, we report the potent synergistic effect of the antidiabetic drug metformin in combination with TIG against tet(A) mutant and tmexCD1-toprJ1 positive K. pneumoniae. The fractional inhibitory concentration index (FICI) of TIG and metformin were less than 0.05 for all the tested isolates. The time-kill curve assay showed that the combination of TIG and metformin exhibited much better antimicrobial effect than TIG alone. The synergistic effect was also confirmed in vivo using a well-studied Galleria mellonella larvae model. Mechanistic studies demonstrated that metformin disrupted the important component of proton motive force, the electric potential $(\Delta \psi)$ and the function of efflux pump, thereby increasing the intracellular concentration of TIG. This finding revealed that metformin might be a possible adjuvant of TIG for combating with superbugs carrying the tet(A) mutant and tmexCD1-toprJ1 genes.

Keywords: tigcycline; metformin; tmexCD1-toprJ1; tet(A); synergy

\section{Introduction}

Antimicrobial resistance (AMR) is a clear and present crisis to "one health". Right now, at least 700,000 people succumb to AMR every year [1]. If no intervening measures are made, by 2050, 10 million patients would die and a cumulative 100 trillion dollars would be generated as a result of AMR [2]. In the last few years, plasmid-mediated resistance genes encoding New Delhi metallo-beta-lactamase (NDM) [3] and phosphoethanolamine transferase enzyme ( $m c r-1)$ [4] that mediate resistance to the clinical last-resort treatments (carbapenems, colistin) were identified and disseminated rapidly worldwide, immensely limited the therapeutic options in clinical practice.

Tigecycline (TIG) is a semi-synthetic parenteral glycylcycline [5]. It not only performs antibacterial effect against tetracycline resistance bacteria, but also shows excellent antibacterial effect against NDM or MCR positive bacteria [6]. TIG has been used in the clinical industry since 2005 [5], and few resistance mechanisms had emerged by 2019. However, recently, a series of resistance genes such as the tet(X3/X4) $[7,8]$, the RND (resistance-nodulation-division) efflux pump gene cluster, tmexCD1-toprJ1 [9], and the tetracycline-efflux pump tet(A) mutation $[10,11]$ were reported to mediate high-level resistance to tigecycline in clinically important pathogens, weakening the efficacy of TIG. 
Worse, a decline in the development of new antibiotics since the 1970s accompanied this AMR crisis [12,13], threatening the convenient therapeutic options in the post-antibiotic era. Therefore, it is urgent to rescue the efficiency of TIG against tet(X3/X4), tmexCD1-toprJ1 and tet(A) mutant positive pathogens.

The antibiotic adjuvant strategy is a cost-effective and promising approach to extend the lifespan of existing antibiotics through inhibiting bacterial resistance or enhancing antibiotic killing [14]. It was reported that the non-steroidal anti-inflammatory drug benzydamine reversed tmexCD-toprJ-mediated TIG resistance [15]. In our previous study, we found that the antidiabetic drug metformin showed no synergy effect with TIG against tet(X3/X4)-positive bacteria, but exhibited the potentiation effect on doxycycline and minocycline against MDR Gram-positive and -negative pathogens through disrupting membrane potential as well as outer membrane permeabilization, and inhibiting the functions of efflux pump [16]. As both the tmexCD1-toprJ1 and tet(A) mutant mediate TIG resistance via efflux pump, we hypothesize whether the metformin could restore the efficacy of TIG against tmexCD1-toprJ1 and tet(A) mutant positive Klebsiella pneumoniae (K. pneumoniae).

In this study, we sought to extend the synergistic activity of metformin in combination with TIG against MDR K. pneumoniae harbouring tmexCD1-toprJ1 or tet(A) mutant. We found that metformin significantly potentiated the antibacterial activity of TIG against tmexCD-toprJ-and tet (A)- bearing bacteria, both in vitro and in the Galleria mellonella infection model. The potentiation of metformin to TIG is attributed to the dysfunction of the efflux pump and increasing the intracellular accumulation of TIG. Our results demonstrate that the antidiabetic drug metformin is a potent antibacterial adjuvant in conjunction with TIG for the treatment of infection caused by tet(A) mutant and tmexCD1-toprJ1 positive K. pneumoniae.

\section{Materials and Methods}

\subsection{Bacteria and Reagents}

Two tmexCD1-toprJ1 positive K. pneumoniae (RGT9-1, RGF15-2-1) and one tet(A) mutant positive K. pneumoniae RGF131 were isolated from swine faeces in 2020 by our lab. All the isolates mediate high level resistance to tigecycline (TIG) with the MIC value over than $16 \mu \mathrm{g} / \mathrm{mL}$. The TIG, ciprofloxacin, meropenem, gentamicin, and metformin were purchased from Yuanye Biological Technology Company (Shanghai, China). Other fluorescence probes and reagents were purchased from Sigma-Aldrich (Saint Louis, MO, USA).

\subsection{MIC Determination}

The minimum inhibitory concentration (MIC) of TIG, ciprofloxacin, gentamicin, and metformin against K. pneumoniae RGT9-1, RGF15-2-1, and RGF131 were determined by the broth micro-dilution method according to the guidance of the Clinical and Laboratory Standards Institute (CLSI) and interpreted in accordance with the CLSI standard [17]. E. coli ATCC 25922 was used as the quality control strain.

\subsection{Checker Board Assay}

Synergistic activity between TIG or other antibiotics (ciprofloxacin, gentamicin, and meropenem) and metformin were measured by the well-studied checkerboard assay [18]. Briefly, $100 \mu \mathrm{L}$ MHB was dispensed into a 96-well micro-titer plate, and then the metformin and TIG (or ciprofloxacin, gentamicin, meropenem) were serially diluted seven times to reach an $8 \times 8$ matrix. Then, $100 \mu \mathrm{L}$ fresh bacterial suspensions at a concentration of $10^{6} \mathrm{cfu} / \mathrm{mL}$ were added. The mixture was incubated at $37^{\circ} \mathrm{C}$ for $18 \mathrm{~h}$, and then the optical density (OD) value at $600 \mathrm{~nm}$ was measured by the Microplate reader (Tecan, Männedorf, Switzerland). The synergistic activity was interpreted with the fractional inhibitory concentrations index (FICI), that was calculated according to the following formula: FICI = FICA + FICB = MICAB $/ \mathrm{MICA}+\mathrm{MICBA} / \mathrm{MICB}$ [18]. The FICA and FICB are the FIC index of drug A and B, respectively; MICA and MICB are the MIC of drug A 
and $\mathrm{B}$, respectively; and MICAB and MICBA are the MIC of one drug in combination with another. Synergism was defined when the FICI is equal to or less than 0.5.

\subsection{Time-Killing Curve}

Overnight culture of each isolate was diluted 1:10,000 into fresh $\mathrm{MH}$ broth and incubated for $6 \mathrm{~h}$ at $37^{\circ} \mathrm{C}$ under continuous shaking (200 rpm). Then, the culture was treated with TIG or metformin alone or their combination for $24 \mathrm{~h}$. At the time points $0,4,8,12$, and $24 \mathrm{~h}$, bacterial numbers were calculated with plate colony counting method. The concentrations of $16 \mu \mathrm{g} / \mathrm{mL}$ TIG and $50 \mu \mathrm{g} / \mathrm{mL}$ metformin was used for K. pneumoniae RGF131 while $32 \mu \mathrm{g} / \mathrm{mL}$ TIG and $25 \mu \mathrm{g} / \mathrm{mL}$ metformin was used for K. pneumoniae RGF15-2-1 and RGT9-1. MH broth with PBS were used as a negative control. Each experiment was performed with three biological replicates.

\subsection{The Proton Motive Force Assay}

The important component of proton motive force (PMF), the electric potential $(\Delta \psi)$ of all the three isolates treated by metformin was measured with fluorescence probe $3,3^{\prime}$ dipropylthiadicarbocyanine iodide (DiSC3(5)) as described in our previous study [15]. Bacterial cells were washed and resuspended to obtain an OD600 of 0.5 with PBS. Additionally, then DiSC3(5) (Aladdin, Shanghai, China) was added at a final concentration of $5 \mu \mathrm{M}$. After $30 \mathrm{~min}$ incubation, $180 \mu \mathrm{L}$ samples were transferred in a black-walled plate and fluorescence was measured immediately in an Infinite M200 Microplate reader (Tecan) with excitation wavelength at $622 \mathrm{~nm}$ and emission wavelength at $670 \mathrm{~nm}$ with an interval of $2 \mathrm{~min}$ for $34 \mathrm{~min}$. Metformin was added at the time point of $4 \mathrm{~min}$ with a final concentration of 10 and $20 \mu \mathrm{g} / \mathrm{mL}$.

\subsection{Efflux Pump Assay}

The effect of metformin on the function of tmexCD1-toprJ1- and tet(A)-mediated efflux pumps was evaluated with the fluorescence dye Rhodamine B [19]. The same cells as in the time-killing curve assay were washed with PBS three times and resuspended with PBS to attain a final OD of 0.5 . A final concentration of Rhodamine B ( $5 \mu \mathrm{M}$ for K. pneumoniae RGF15-2-1, RGT9-1 and $50 \mu \mathrm{M}$ for K. pneumoniae RGF131) was added. Then, the mixture was cultured for $30 \mathrm{~min}$ at $37^{\circ} \mathrm{C}$ under continuous shaking (200 rpm). The extra Rhodamine $\mathrm{B}$ were washed with PBS three times, then the pellets were re-suspended in PBS containing $1 \%$ glucose. Metformin at a final concentration of 25,50 , and $100 \mathrm{mg} / \mathrm{mL}$ was then added and incubated at $37^{\circ} \mathrm{C}$ for $30 \mathrm{~min}$. Finally, the bacteria were centrifuged at $4000 \times g$ for 10 min and then Rhodamine B efflux from the cells was monitored with the excitation wavelength at $540 \mathrm{~nm}$ and emission wavelength at $625 \mathrm{~nm}$ using Infinite M200 Microplate reader.

\subsection{Tigecycline Intracellar Accumulation Analysis}

The well-studied high-performance liquid chromatography-tandem mass spectrometry (HPLC-MS/MS) method for the determination of TIG was used to determine the accumulation of TIG in K. pneumoniae RGF15-2-1 and K. pneumoniae RGF131 [20]. Briefly, $1.0 \mathrm{~mL}$ of an overnight culture of each isolate was diluted into $100 \mathrm{~mL}$ fresh Luria Bertani (LB) broth and grown at $37^{\circ} \mathrm{C}$ under continuous shaking (200 rpm) to an OD600 of 0.5. Then, bacteria cells were pelleted and diluted to $10^{10} \mathrm{cfu} / \mathrm{mL}$ with PBS. TIG at MIC concentration together with varying metformin were added, then samples were incubated at $37{ }^{\circ} \mathrm{C}$ under continuous shaking $(200 \mathrm{rpm})$ for $15 \mathrm{~min}$. The extracellular drug was discarded by centrifuging at $12,000 \times \mathrm{g}$ for $10 \mathrm{~min}$. The pretreatment method for drug extraction was identical with our previous report [16]. To lyse the cells, an aliquot of $500 \mu \mathrm{L}$ water was added to the pellet, and then subjected to three freeze-thaw cycles in liquid nitrogen followed by water bath at $55^{\circ} \mathrm{C}$. The supernatant was collected via centrifugation at $12,000 \times g$ for $10 \mathrm{~min}$. Additionally, another $500 \mu \mathrm{L}$ acetonitrile were added to the pellet 
to further extract the TIG and pelleted again. The supernatants were combined and filtered with a $0.22 \mu \mathrm{m}$ filter membrane.

Finally, the supernatants were detected by an Agilent 1260 Infinity HPLC system combined with AB SCIEX QTRAP 6500 mass spectrometer (ABSciex, Foster City, CA, USA). The TIG was separated on a C18 column. The mobile phase was a solution of Millipore water $(0.2 \% v / v$ formic acid) $(\mathrm{A})$ and $\mathrm{ACN}(\mathrm{B})$ with a gradient elution as follows: $0-0.5 \mathrm{~min}$, 90\% A; 0.5-1.5 min, 90-10\% A; 1.5-5.0 min, 10\% A; 5.0-5.5 min, 10-90\% A; and 5.5-7.0 min, $90 \%$ A. The quantification detection of TIG was analysed by multiple reaction monitoring $(\mathrm{MRM})$ with positive electrospray ionization using the $\mathrm{m} / \mathrm{z} 586.4 \rightarrow 513.3$ transition.

\subsection{Galleria Mellonella Infection Model}

Galleria mellonella larvae was supplied by Huiyude Biotech Company (Tianjin, China). They were divided into 12 groups ( $n=10$ per group). All larvae were infected with $10^{7} \mathrm{CFUs}$ K. pneumoniae RGT9-1, RGF15-2-1 or RGF131 suspension, respectively. At $1 \mathrm{~h}$ post-infection, infected larvae were treated with PBS, TIG (50 mg/ $\mathrm{kg}$ b.w.), metformin (50 mg/kg b.w.), or the combination of TIG with metformin $(25+25 \mathrm{mg} / \mathrm{kg}$ b.w.). The survival rates of each group were recorded for 5 days.

\subsection{Data Analysis}

All data are expressed as mean \pm SD from three biological replicates. Statistical significance was determined using Graphpad Prism 7.0 software with unpaired Student's $t$-test or non-parametric one-way ANOVA.

\section{Results}

3.1. The In Vitro Synergistic Activity of Tigecycline and Metformin against tmexCD1-toprJ1 and tet(A) Mutant Positive Strains

The MIC results of antibiotics and metformin were determined using the CLSI recommended broth micro-dilution method. The results are shown in Table 1 . Both the tmexCD1-toprJ1 positive strains (RGF15-2-1 and RGT9-1) exhibited high resistance to TIG, tetracycline, ciprofloxacin, and gentamicin, but were susceptible to meropenem. The tet(A) mutant isolate was resistance to TIG and tetracycline and susceptible to meropenem. The MIC of metformin against all the three isolates were $50 \mathrm{mg} / \mathrm{mL}$.

Table 1. Basic information and susceptibility profiles of K. pneumoniae harbouring tmexCD1-toprJ1 or tet(A) mutation.

\begin{tabular}{|c|c|c|c|c|c|c|c|c|c|c|c|c|c|}
\hline \multirow{2}{*}{ Strain } & \multirow{2}{*}{ Resistance Gene } & \multirow{2}{*}{ Sources } & \multicolumn{11}{|c|}{ MIC $(; \mu \mathrm{g} / \mathrm{mL})$} \\
\hline & & & GEN & CFF & CIP & ENR & TET & TGC & DOX & MET & MER & CL & KAN \\
\hline RGT9-1 & $\begin{array}{l}\text { tmexCD1-toprJ1,oqxAB, QnrB4, QnrS1, } \\
\text { aac(3)-IId, aac (6')Ib-cr, aadA16, aadA2 }\end{array}$ & $\begin{array}{l}\text { Swine } \\
\text { Faeces }\end{array}$ & 128 & 8 & 8 & 8 & $>128$ & 64 & 64 & $50 \mathrm{mg}$ & $\leq 0.25$ & $\leq 0.25$ & $>256$ \\
\hline RGF15-2-1 & $\begin{array}{l}\text { tmexCD1-toprJ1,oqxAB, QnrB4, QnrS1, } \\
\text { aac(3)-IId, aac(6')Ib-cr, aadA16, aadA2 }\end{array}$ & $\begin{array}{l}\text { Swine } \\
\text { Faeces }\end{array}$ & 128 & 16 & 8 & 8 & $>128$ & 64 & 64 & $50 \mathrm{mg}$ & $\leq 0.25$ & $\leq 0.25$ & $>256$ \\
\hline RGF-131 & $\begin{array}{l}\text { tet(A), QnrS1, aac (3)-IId, aac (6')Ib-cr, aadA16, } \\
\text { aadA1, bla } 6_{C T X-M-55}, \text { bla }_{S H V-11}, \text { bla } a_{S H V-1}\end{array}$ & $\begin{array}{l}\text { Swine } \\
\text { Faeces }\end{array}$ & 64 & 4 & 4 & 4 & $>128$ & 32 & 64 & $50 \mathrm{mg}$ & $\leq 0.25$ & $\leq 0.25$ & $>256$ \\
\hline
\end{tabular}

GEN, gentamicin; CFF, ceftiofur; CIP, ciprofloxacin; ENR, enrofloxacin; TET, tetracycline; TGC, tigecycline; DOX doxycycline; MET, metformin; MER, meropenem; CL, colistin; and KAN, kanamycin.

As metformin exhibits the potentiation effect on doxycycline and minocycline through inhibiting the functions of efflux pump, we tested the synergistic activity of metformin and TIG in fighting with tmexCD1-toprJ1 and tet(A) mutant positive strains with checkboard assays. Notably, obvious synergism was observed for the combination against all three isolates with the FICI of 0.047, 0.047, and 0.030 in K. pneumoniae RGT9-1, RGF15-2-1, and RGF131, respectively (Figure 1A). To further test whether this synergy effect was TIG-specific, the synergistic effects of metformin and other antibacterial (meropenem, ciprofloxacin, and gentamicin) were assessed through checkboard assay. Metformin potentiated the antibacterial effect of ciprofloxacin against tmexCD1-toprJ1 positive K. pneumoniae in fighting with RGF15-2-1 and RGT9-1 with the FICI of 0.14 and 0.16 (Figure 1B). However, 
an antagonistic effect was observed in the combination of ciprofloxacin and metformin in fighting with tet(A) mutant positive K. pneumoniae RGF131 (data not shown). As the resistance to TIG and ciprofloxacin was mediated by tmexCD1-toprJ1 in RGF15-2-1 and RGT9-1 and the tet(A) mutant only mediated resistance to TIG and other tetracyclines in RGF131, we hypothesize metformin inhibited the function of the efflux pump. The antagonism effect was also observed in the combination of metformin and meropenem or gentamicin against RGF15-2-1 and RGT9-1 (Figure 1). Though tmexCD1-toprJ1 mediated resistance to aminoglycoside, there was other resistance gene playing a role in gentamicin resistance; for example, the aminoglycoside adenyltransferase (aadA2) [21]. The results indicate that metformin may potentiate the effect of TIG through inhibit the tmexCD1-toprJ1 and tet(A) efflux pump.

A

K.pneumoniae 9-1
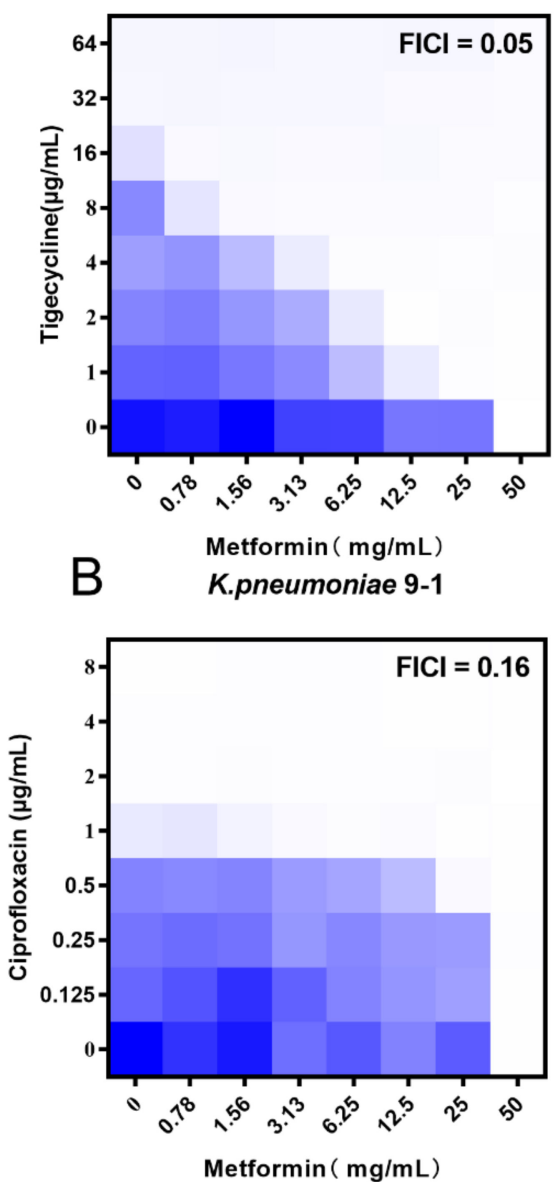

K.pneumoniae 15-2-1

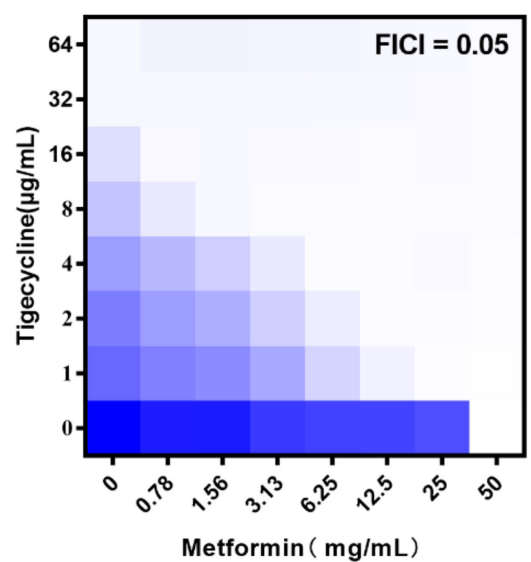

K.pneumoniae 15-2-1

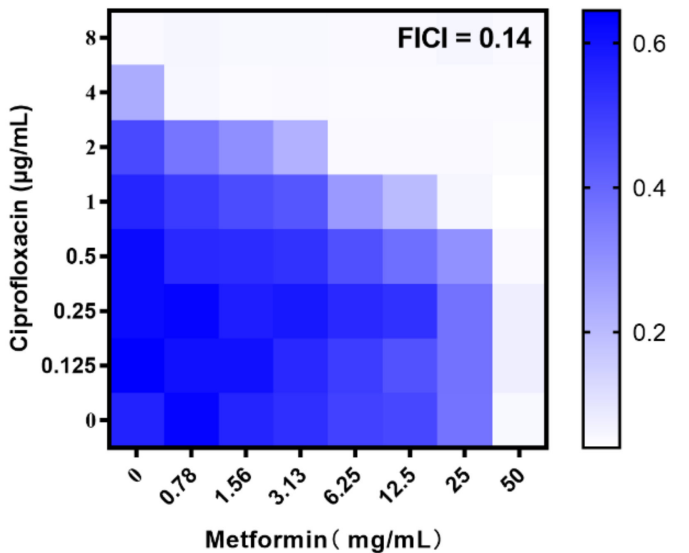

K.pneumoniae 131

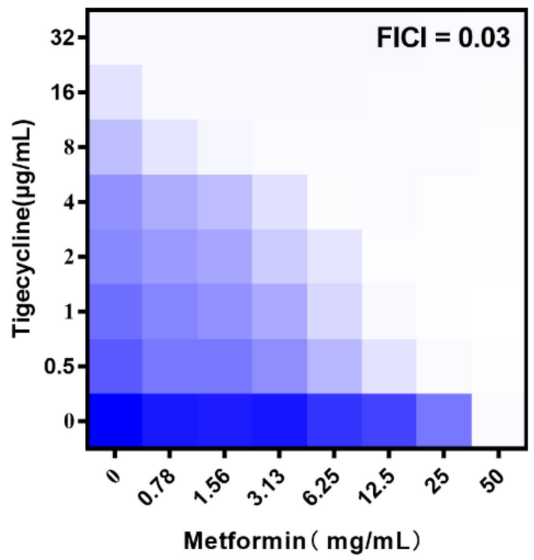

0.2

Figure 1. Synergistic activity of tigecycline and metformin against K. pneumoniae harbouring tmexCD1toprJ1 or tet(A) mutation. (A) Chequerboard broth microdilution assays between metformin and tigecycline against K. pneumoniae. (B) Chequerboard broth microdilution assays between metformin and ciprofloxacin against K. pneumoniae. Dark-blue regions represent higher cell density and lower inhibition rate of combinational treatment. Data represent the mean OD $(600 \mathrm{~nm})$ of two biological replicates. Synergy is defined as an FIC index of $\leq 0.5$.

To further investigate the synergistic effect of TIG and metformin, the time-kill curves were performed for TIG or metformin monotherapy and their combination. We found that either $32 \mu \mathrm{g} / \mathrm{mL}$ TIG (corresponding to MIC) or $25 \mathrm{mg} / \mathrm{mL}$ metformin (corresponding to $1 / 2 \mathrm{MIC}$ ) alone displayed no effect on bactericidal effect. In the contrast, the combination of $32 \mu \mathrm{g} / \mathrm{mL}$ TIG and $25 \mathrm{mg} / \mathrm{mL}$ metformin showed obvious bactericidal activities 
against both RGT9-1 and RGF15-2-1 (Figure 2A,B). The combination effect of TIG and metformin also showed superiority in anti-bacteria effect compared to single drug treatment in the case of tet(A) mutant isolate RGF131 (Figure 2C). Furthermore, the combination of $8 \mu \mathrm{g} / \mathrm{mL}$ ciprofloxacin and $25 \mathrm{mg} / \mathrm{mL}$ metformin showed obvious bactericidal activities against RGF15-2-1 while the single drug cannot even inhibit the growth of RGF15-2-1 (Figure 2D). This results indicated that metformin possesses universal synergistic effect with antibacterial which was inactivated by the tmexCD1-toprJ1 or tet(A) efflux pump.

A

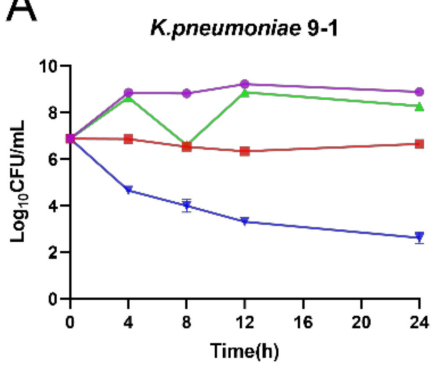

C

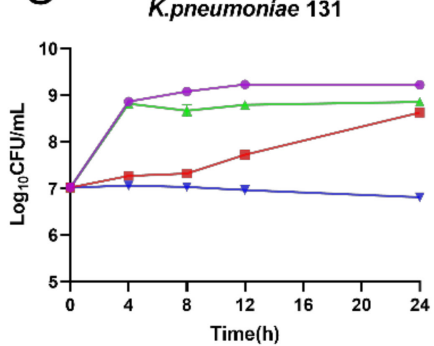

B
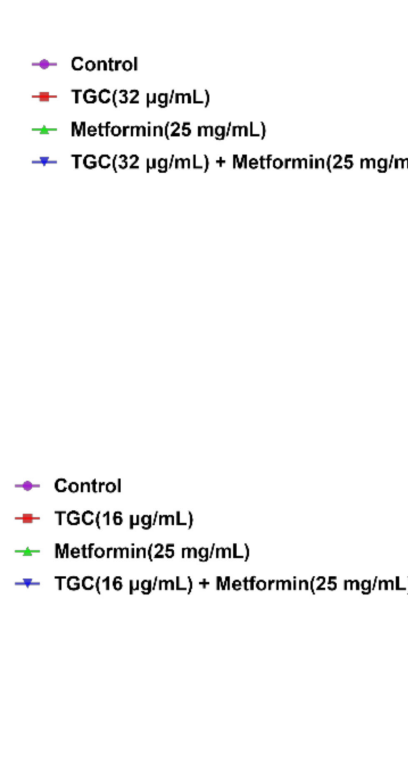

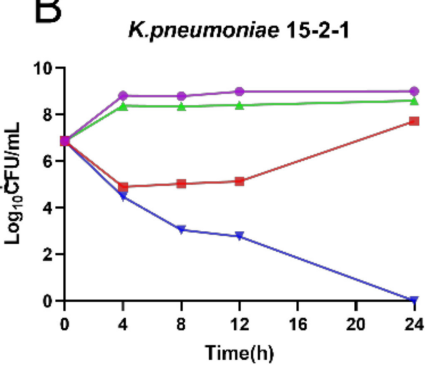

- Control

- TGC(32 $\mu \mathrm{g} / \mathrm{mL})$

- Metformin $(25 \mathrm{mg} / \mathrm{mL})$

* TGC $(32 \mu \mathrm{g} / \mathrm{mL})+$ Metformin $(25 \mathrm{mg} / \mathrm{mL})$

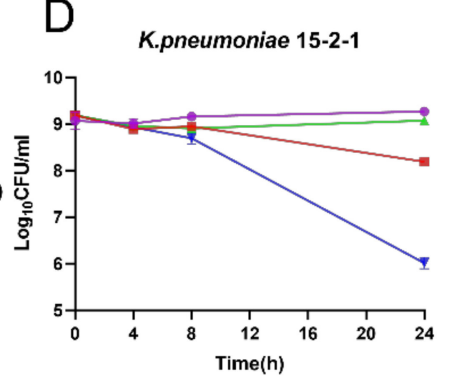

- Control

- $\mathrm{CIP}(8 \mu \mathrm{g} / \mathrm{mL})$

\pm Metformin $(25 \mathrm{mg} / \mathrm{mL})$

* CIP(8 $\mu \mathrm{g} / \mathrm{mL})+$ Metformin $(25 \mathrm{mg} / \mathrm{mL})$

Figure 2. Time-killing curves of the combination of tigecycline and metformin against (A) K. pneumonia 9-1 harbouring tmexCD1-toprJ1; (B) K. pneumoniae 15-2-1harboring tmexCD1-toprJ1; (C) K. pneumoniae 131 harbouring tet(A) mutation; and (D) Time-killing of the combination of ciprofloxacin and metformin against K. pneumonia 15-2-1 harbouring tmexCD1-toprJ1.

\subsection{Metformin Deprives the Function of Efflux Pump and Facilitate the Intracellular Accumulation of Tigecycline}

To elucidate the possible mechanisms of the synergy effect between metformin and TIG, the effect of metformin on the tmexCD1-toprJ1 and tet(A) efflux pump function was investigated. The capability of the tmexCD1-toprJ1 and tet(A) efflux pumps under the treatment of different concentrations of metformin was assessed with a rhodamine-based assay [15]. The effluxes of rhodamine was reduced in a dose-dependent way at the presence of metformin in all the three isolates (Figure $3 \mathrm{~A}-\mathrm{C}$ ).

As the proton motive force (PMF) is essential for the function of efflux pump, we also tested the important component of PMF, the electric potential $(\Delta \psi)$ of all the three isolates treated by metformin with 3,3'-dipropylthiadicarbocyanine iodide (DiSC3(5)) probe. As expected, the addition of metformin at 10 and $20 \mathrm{mg} / \mathrm{mL}$ significantly increased the fluorescence. The increased fluorescence indicated the release of DiSC3(5) from the cytoplasmic membrane to the extracellular milieu due to the disruption of $\Delta \psi$. Thus, the $\Delta \psi$ was disrupted under metformin treatment (Figure 3D-F), as was the PMF.

The intracellular concentration of TIG in all the three isolates after treatment with a series concentration of metformin was measured by HPLC-MS/MS. The concentrations of TIG in bacteria were significant increased in a dose-dependent way at the presence of metformin (Figure 3G,H). The results indicated that TIG was markedly accumulated in bacteria cells under the treatment of metformin. 

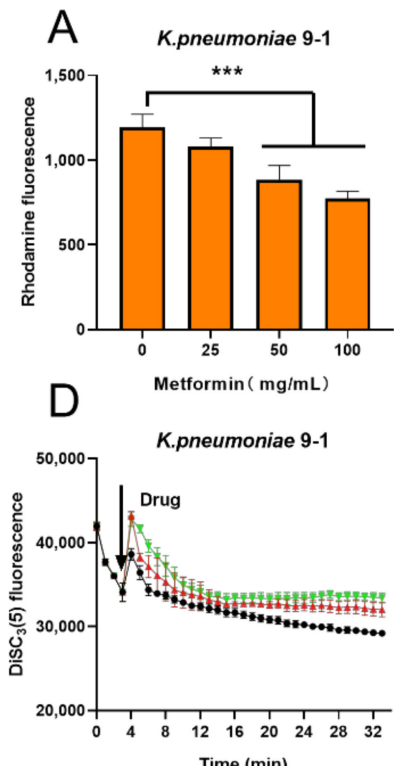

G

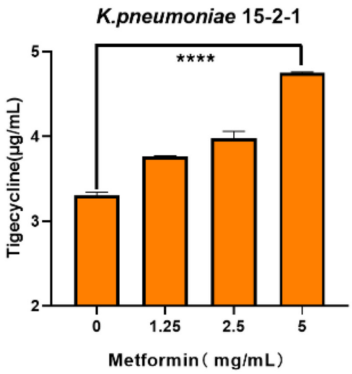

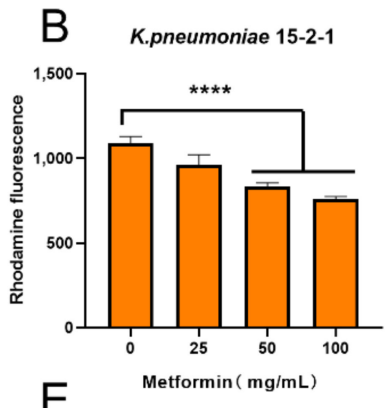
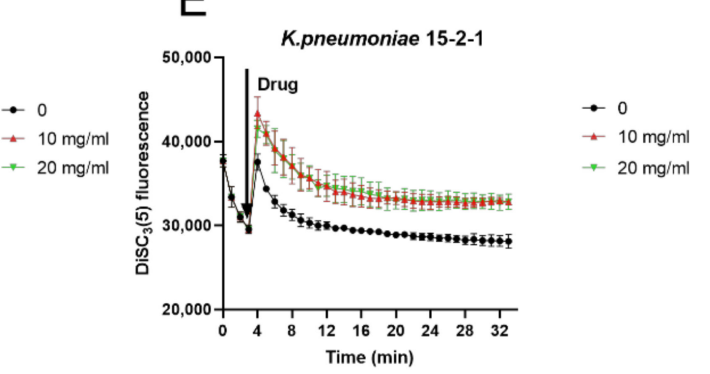

$\mathrm{H}$

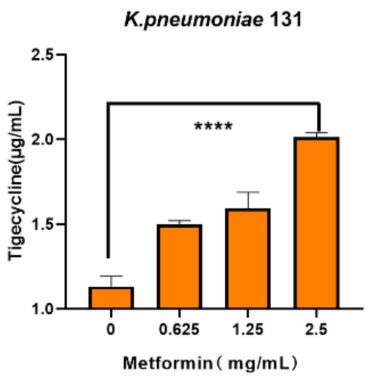

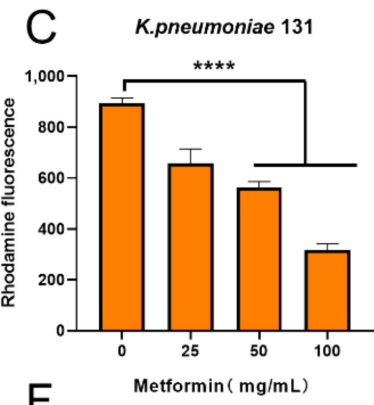

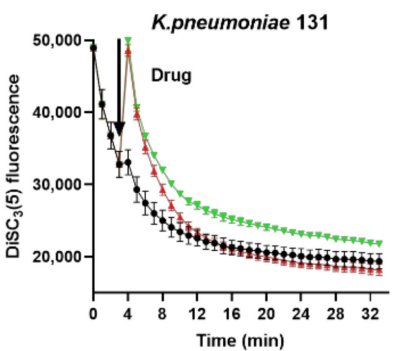

- $10 \mathrm{mg} / \mathrm{ml}$

- $20 \mathrm{mg} / \mathrm{ml}$

Figure 3. Tigecycline-metformin combination exerts synergy by dissipating electric potential $(\Delta \psi)$, destroying the capability of the efflux pump and increase the intracellular concentrations of tigecycline. (A-C) Function of efflux pump of (A) K. pneumonia 9-1 harbouring tmexCD1-toprJ1; (B) K. pneumoniae 15-2-1harboring tmexCD1-toprJ1; and (C) K. pneumoniae harbouring tet(A) mutation after exposure to varying concentrations of metformin, measured by the fluorescence dye Rhodamine. (D-F) Membrane potential changes in (D) K. pneumonia 9-1 harbouring tmexCD1-toprJ1; (E) K. pneumoniae 15-2-1 harbouring tmexCD1-toprJ1; and (F) K. pneumoniae harbouring tet(A) mutation upon exposure to metformin, probed by potentiometric fluorophore DiSC3(5). (G,H) Intracellular accumulation of tigecycline in cells treated with metformin determined by HPLC-MS/MS analysis. Initial concentration of tigecycline was $32 \mu \mathrm{g} / \mathrm{mL}$. All data are expressed as mean $\pm \mathrm{SD}$ from three biological replicates and $p$ values were determined by non-parametric one-way ANOV A $\left({ }^{* * *} p<0.001\right.$, **** $p<0.0001)$.

\subsection{Metformin Potentiates In Vivo Efficacy of Tigecycline Using Galleria Mellonella Infection Model}

After confirming the in vitro synergistic activity of TIG and metformin against tmexCD1toprJ1 and tet $(A)$ mutant positive strains, we further assessed whether these synergy effects could be observed in vivo. To confirm this, a well-studied preclinical infection model (Galleria mellonella larvae infection models) was constructed with all the three isolates. The larvae in blank control all died in $72 \mathrm{~h}$, except the strain of RGF15-2-1. The survival rates in TIG or metformin monotherapy groups were below $25 \%$ for all the isolates. In contrast, the survival rates in combination groups were significantly increased with the $p$ values of $0.002,0.010$, and 0.029 compared to TIG monotherapy group for infection of K. pneumoniae RGT9-1, RGF15-2-1, or RGF131, respectively (Figure 4A-C). It should be stressed here that the dose in combination group was just half that in monotherapy groups. The in vivo 
results demonstrated the adjuvant potential of metformin with TIG in fight with infectious diseases caused by tmexCD1-toprJ1 and tet(A) mutant positive pathogens.

A

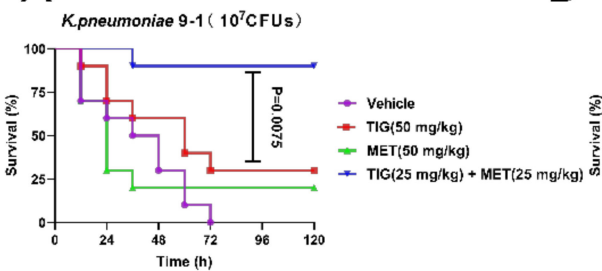

B

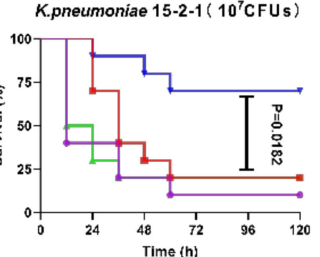

C

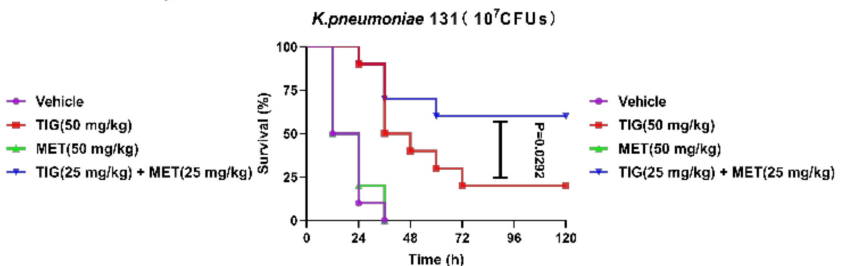

Figure 4. Metformin effectively improves tigecycline efficacy in Galleria mellonella infection model. Survival rates of Galleria mellonella larvae ( $n=10$ per group) infected by (A) K. pneumoniae 9-1 harbouring tmexCD1-toprJ1; (B) K. pneumoniae 15-2-1 harbouring tmexCD1-toprJ1; and (C) K. pneumoniae harbouring tet(A) mutation after treatment with PBS as vehicle, a single dose of metformin (MET, $50 \mathrm{mg} / \mathrm{kg}$ ), tigecycline (TIG, $50 \mathrm{mg} / \mathrm{kg}$ ), or their combination with concentration of $25 \mathrm{mg} / \mathrm{kg}$, respectively. $p$ values were determined by log-rank (Mantel-Cox) test.

\section{Discussion}

TIG was the last antibiotic choice in fighting carbapenems and colistin resistance Gram-negative pathogen infections. However, with the identification and spreading of the genetic elements tet (X3/X4), tmexCD1-toprJ1, and tet(A) mutant mediating high levels of TIG resistance, the effect of TIG was much weakened $[8,9,11]$. Fortunately, the adjuvant strategy, which restores antibiotic efficacy against MDR pathogen infection, was prosperous in recent years. For example, thioridazine restores the antibacterial effect of oxacillin against MRSA [22]; a synergy effect was observed between Phenothiazines and Oxacillin against MRSA [23]; and anti-HIV agent azidothymidine decreases tet $(X)$ mediated bacterial resistance to tigecycline in Escherichia coli (E. coli) [24]. These exciting results inspire us to find more potential adjuvants of the last restored drug TIG against super bugs. In our previous study, we found that an FDA-approved hypoglycemic drug metformin potentiates tetracyclines (except TIG) against multiple tet(A) positive pathogens including S. aureus, vancomycin-resistant enterococci, E. coli, and S. enteritidis [16]. We speculated that the low level of TIG resistance mediated by tet(A) may account for the no synergy effect between metformin and TIG. Thus, in this study, we sought to extend the synergistic activity of metformin in combination with TIG against K. pneumoniae harbouring tmexCD1-toprJ1 or tet(A) mutant. Interestingly, synergistic activity was observed between metformin and TIG against tet(A) mutant positive K. pneumoniae RGF131 with the FICI of 0.03 . It is worth noting that the tet(A) mutant mediated high level resistance to TIG with the MIC value of $32 \mu \mathrm{g} / \mathrm{mL}$ in K. pneumonia RGF131. In the meantime, metformin significantly potentiated the antibacterial activity of TIG against tmexCD1-toprJ1 bearing bacteria, which mediated resistance against multiple kinds of antimicrobials such as tetracyclines and fluoroquinolones. On the other hand, synergy was also observed between metformin and ciprofloxacin against tmexCD1-toprJ1 bearing bacteria, indicating metformin was a universal adjuvant against tmexCD1-toprJ1 positive bacteria. The time-kill curve study not only verified the synergistic activity of metformin in combination with TIG or ciprofloxacin, but also displayed a time-dependent bactericidal activity of the combinations, highlighting the potency of the drug combination. The in vivo synergism effect of metformin and TIG against all three isolates were assessed and verified using a well-studied Galleria mellonella infection model. It is a pity that the adjuvant therapy effect of this combination was not tested in a rodent disease model in this study. As the adjuvant potential of metformin with doxycycline was obvious in a mouse peritonitis infection model infected with E. coli B2, the in vivo synergism effect of metformin and TIG was expected in rodent disease model. However, further study is needed to confirm it.

It was reported in our previous study that metformin potentiated doxycycline antibacterial effects through disrupting membrane potential $(\Delta \psi)$ [16]. Thus, the $\Delta \psi$ was 
assessed in the presence of metformin. As expected, the $\Delta \psi$ was significantly disrupted. It was reported that the $\Delta \psi$ is an important component of PMF [25]. Additionally, the PMF was essential for the function of efflux pump [26]. The dissemination of PMF might affect the capability of the efflux pump. Interestingly, the activity of the efflux function of tmexCD1-toprJ1 and tet(A) mutant was weakened in a dose-dependent manner by metformin in the rhodamine assay. Similarly, the intracellular concentrations of TIG were markedly increased under the action of metformin. As TIG only exhibits antibacterial activity when a sufficient drug enters into bacteria cells and binds to the $30 \mathrm{~S}$ ribosomal subunit [27], intracellular TIG concentration is important for the antibacterial effects of TIG. Metformin disrupted the PMF, hence blocking the tmexCD1-toprJ1 and tet(A) efflux pumps and promoting the accumulation of TIG in cells. Thus, the potentiation of metformin to TIG is attributed to the dysfunction of the efflux pump and increasing the intracellular accumulation of TIG. These findings demonstrate that antidiabetic drug metformin is a potent antibacterial adjuvant in conjunction with TIG for the treatment of infection caused by tet(A) mutant and tmexCD1-toprJ1 positive K. pneumoniae.

Author Contributions: X.X. (Xia Xiao) and Z.W. conceived and designed the project; Q.H., Y.H., Y.L. and R.L. performed all experiments; X.X. (Xilan Xu), Y.L. and R.L. analysed the data; and X.X. (Xia Xiao) and Q.H. wrote the manuscript. All authors have read and agreed to the published version of the manuscript.

Funding: This work was supported by the National Natural Science Foundation of China (31702291, 31872526) and A Project Funded by the Priority Academic Program Development of Jiangsu Higher Education Institutions (PAPD).

Institutional Review Board Statement: Not applicable.

Informed Consent Statement: Not applicable.

Data Availability Statement: Data is contained within the article.

Conflicts of Interest: The authors declare no conflict of interest.

\section{References}

1. Majumder, A.A.; Rahman, S.; Cohall, D.; Bharatha, A.; Singh, K.; Haque, M.; Hilaire, M.G.-S. Antimicrobial Stewardship: Fighting Antimicrobial Resistance and Protecting Global Public Health. Infect. Drug Resist. 2020, 13, 4713-4738. [CrossRef]

2. Mullard, A. An audience with ... Jim O'Neill. Nat. Rev. Drug Discov. 2016, 15, 526. [CrossRef]

3. Walsh, T.R.; Weeks, J.; Livermore, D.M.; Toleman, M.A. Dissemination of NDM-1 positive bacteria in the New Delhi environment and its implications for human health: An environmental point prevalence study. Lancet Infect. Dis. 2011, 11, 355-362. [CrossRef]

4. Liu, Y.-Y.; Wang, Y.; Walsh, T.R.; Yi, L.-X.; Zhang, R.; Spencer, J.; Doi, Y.; Tian, G.; Dong, B.; Huang, X.; et al. Emergence of plasmid-mediated colistin resistance mechanism MCR-1 in animals and human beings in China: A microbiological and molecular biological study. Lancet Infect. Dis. 2016, 16, 161-168. [CrossRef]

5. Pankey, G.A. Tigecycline. J. Antimicrob. Chemother. 2005, 56, 470-480. [CrossRef]

6. Rodriguez-Bano, J.; Gutiérrez-Gutiérrez, B.; Machuca, I.; Pascual, A. Treatment of Infections Caused by Extended-Spectrum-BetaLactamase-, Ampc-, and Carbapenemase-Producing Enterobacteriaceae. Clin. Microbiol. Rev. 2018, 31, e00079-17. [CrossRef] [PubMed]

7. He, T.; Wang, R.; Liu, D.; Walsh, T.; Zhang, R.; Lv, Y.; Ke, Y.; Ji, Q.; Wei, R.; Liu, Z.; et al. Emergence of plasmid-mediated high-level tigecycline resistance genes in animals and humans. Nat. Microbiol. 2019, 4, 1450-1456. [CrossRef]

8. Sun, J.; Chen, C.; Cui, C.-Y.; Zhang, Y.; Liu, X.; Cui, Z.-H.; Ma, X.-Y.; Feng, Y.-J.; Fang, L.-X.; Lian, X.-L.; et al. Plasmid-encoded tet $(\mathrm{X})$ genes that confer high-level tigecycline resistance in Escherichia coli. Nat. Microbiol. 2019, 4, 1457-1464. [CrossRef]

9. Lv, L.; Wan, M.; Wang, C.; Gao, X.; Yang, Q.; Partridge, S.R.; Wang, Y.; Zong, Z.; Doi, Y.; Shen, J.; et al. Emergence of a Plasmid-Encoded Resistance-Nodulation-Division Efflux Pump Conferring Resistance to Multiple Drugs, Including Tigecycline, in Klebsiella pneumoniae. mBio 2020, 11, 2930. [CrossRef]

10. Chiu, S.K.; Huang, L.Y.; Chen, H.; Tsai, Y.K.; Liou, C.H.; Lin, J.C.; Siu, L.K.; Chang, F.Y.; Yeh, K.M. Roles of ramR and tet(A) Mutations in Conferring Tigecycline Resistance in Carbapenem-Resistant Klebsiella pneumoniae Clinical Isolates. Antimicrob. Agents Chemother. 2017, 61, e00391-17. [CrossRef]

11. Xu, J.; Zhu, Z.; Chen, Y.; Wang, W.; He, F. The Plasmid-Borne tet(A) Gene Is an Important Factor Causing Tigecycline Resistance in ST11 Carbapenem-Resistant Klebsiella pneumoniae Under Selective Pressure. Front. Microbiol. 2021, 12, 644949. [CrossRef]

12. Vila, J.; Moreno-Morales, J.; Ballesté-Delpierre, C. Current landscape in the discovery of novel antibacterial agents. Clin. Microbiol. Infect. 2020, 26, 596-603. [CrossRef] [PubMed] 
13. Theuretzbacher, U.; Outterson, K.; Engel, A.; Karlén, A. The global preclinical antibacterial pipeline. Nat. Rev. Genet. 2019, 18, 275-285. [CrossRef] [PubMed]

14. Gupta, V.; Datta, P. Next-generation strategy for treating drug resistant bacteria: Antibiotic hybrids. Indian J. Med. Res. 2019, 149, 97-106. [PubMed]

15. Tong, Z.; Xu, T.; Deng, T.; Shi, J.; Wang, Z.; Liu, Y. Benzydamine Reverses tmexCD-toprJ-Mediated High-Level Tigecycline Resistance in Gram-Negative Bacteria. Pharmaceuticals 2021, 14, 907. [CrossRef]

16. Liu, Y.; Jia, Y.; Yang, K.; Li, R.; Xiao, X.; Zhu, K.; Wang, Z. Metformin Restores Tetracyclines Susceptibility against Multidrug Resistant Bacteria. Adv. Sci. 2020, 7, 1902227. [CrossRef]

17. CLSI. Clinical \& Laboratory Standards Institute, Performance Standards for Antimicrobial Susceptibility Testing: Twenty-Fourth Informational Supplement M100-S24; CLSI: Wayne, PA, USA, 2014.

18. Odds, F.C. Synergy, antagonism, and what the chequerboard puts between them. J. Antimicrob. Chemother. 2003, 52, 1. [CrossRef]

19. Forster, S.; Thumser, A.E.; Hood, S.R.; Plant, N. Characterization of Rhodamine-123 as a Tracer Dye for Use In In vitro Drug Transport Assays. PLoS ONE 2012, 7, e33253. [CrossRef]

20. Munyeza, C.F.; Shobo, A.; Baijnath, S.; Bratkowska, D.; Naiker, S.; Bester, L.A.; Singh, S.D.; Maguire, G.E.M.; Kruger, G.; Naicker, T.; et al. Development and validation of a liquid chromatography-tandem mass spectrometry (LC-MS/MS) method for the quantification of tigecycline in rat brain tissues. Biomed. Chromatogr. 2015, 30, 837-845. [CrossRef]

21. Ahmed, A.M.; Shimamoto, T. A Plasmid-Encoded Class 1 Integron Carrying Sat, a Putative Phosphoserine Phosphatase Gene and Aada2 from Enterotoxigenic Escherichia Coli O159 Isolated in Japan. FEMS Microbiol. Lett. 2004, 235, 243-248. [CrossRef]

22. Bonde, M.; Højland, D.H.; Kolmos, H.J.; Kallipolitis, B.H.; Klitgaard, J.K. Thioridazine affects transcription of genes involved in cell wall biosynthesis in methicillin-resistant Staphylococcus aureus. FEMS Microbiol. Lett. 2011, 318, 168-176. [CrossRef] [PubMed]

23. Ding, X.; Yang, C.; Moreira, W.; Yuan, P.; Periaswamy, B.; De Sessions, P.F.; Zhao, H.; Tan, J.; Lee, A.; Ong, K.X.; et al. A Macromolecule Reversing Antibiotic Resistance Phenotype and Repurposing Drugs as Potent Antibiotics. Adv. Sci. 2020, 7, 2001374. [CrossRef] [PubMed]

24. Liu, Y.; Jia, Y.; Yang, K.; Li, R.; Xiao, X.; Wang, Z. Anti-HIV agent azidothymidine decreases tet(X)-mediated bacterial resistance to tigecycline in Escherichia coli. Commun. Biol. 2020, 3, 162. [CrossRef] [PubMed]

25. Glasser, N.R.; Kern, S.E.; Newman, D.K. Phenazine redox cycling enhances anaerobic survival inPseudomonas aeruginosaby facilitating generation of ATP and a proton-motive force. Mol. Microbiol. 2014, 92, 399-412. [CrossRef] [PubMed]

26. Webber, M.A.; Piddock, L.J. The importance of efflux pumps in bacterial antibiotic resistance. J. Antimicrob. Chemother. 2003, 51, 9-11. [CrossRef]

27. Chopra, I.; Roberts, M. Tetracycline Antibiotics: Mode of Action, Applications, Molecular Biology, and Epidemiology of Bacterial Resistance. Microbiol. Mol. Biol. Rev. 2001, 65, 232-260. [CrossRef] 